\title{
Prevalence of dengue virus infection among population of Bhaili visiting tertiary health institution; Chhattisgarh
}

\author{
Dhruba Hari Chandi \\ Assistant Professor, Department of Microbiology, Jawaharlal Nehru Medical College, Sawangi(M), Wardha, Maharashtra
}

Background: Dengue fever, dengue haemorrhagic fever and dengue shock syndrome of infection is distinct clinical forms which is caused by Dengue Virus that belong to a member of the Flaviviridae family. It is most important arthropod borne viral disease that causes morbidity and mortality. Dengue virus infection is a major public health problem growing in worldwide and it is estimated about 2.5 billion people of world are at risk of this infection. In India several parts of the country it is an endemic disease. Aims and Objective: The current work is an attempt to review current perspectives of dengue infection among population of Bhilai visiting tertiary health institution. In study also we have compared the serological profiles of the dengue cases. Materials and Methods: Samples received were performing retrospective analysis in the department of microbiology and process in the departmental microbiology laboratory obtaining during monsoon season. The duration of fever (in days) and other relevant clinical information were recorded from the requisition form. Rapid test for dengue as NS1 Ag, IgG and IgM test were performed in accordance with the manufacturer's instructions. The dengue NS1 Ag, IgG and IgM rapid test is an in vitro immunochromatographic test (ICT) which is a onestep assay designed for the qualitative determination of dengue NS1 Ag, IgG and IgM in human serum for the diagnosis dengue infection. Result: During the study period total 1308 serum samples were collected from suspected Dengue fever patients. Among 1308 samples $412(31.5 \%)$ samples were found to be positive dengue fever. In the month of August maximum number of sample were received. Out of 1308 patients with dengue suspected patients $792(60.6 \%)$ were male and $516(39.4 \%)$ were female. Among $60.6 \%$ of male $28.2 \%$ were positive and out of $39.4 \%$ of female $36.6 \%$ were positive for dengue. Age between $10-20$ years old $(55.83 \%)$ patients were most affected age group and $70-80$ years old $(8.77 \%)$ age group were least affected. Among the dengue positive patients, dengue parameter associated with thrombocytopenia was also recorded which showed that there was no significant difference between the parameters in relation to thrombocytopenia. Conclusion: Especially in developing countries like India where there are poor resources for diagnosis of dengue infection, the sensitivity of these tests is more than immunochromatographic Test (ICT). By NS1 assay early detection of dengue infection can help in early confirmation and management of this before its gets complicate. Therefore commercial available dengue NS1 antigen test kits provided additional laboratory diagnostic tool for early detection of dengue.

Key words: Prevalence; Dengue; Thrombocytopenia; NS1; IgM; IgG

\section{INTRODUCTION}

From long period of history to this era, present information about public health news is uncommon pathogens or infection syndromes; infection of dengue virus (family Flaviviridae) is an endemic problem increasingly important in many parts of the world. Dengue fever is a mosquito born viral disease which is widely spread in tropical and sub tropical regions of the world. Dengue virus infection is a major public health 
problem growing in worldwide and it is estimated about 2.5 billion people of world are at risk of this infection. In India several parts of the country it is an endemic disease. Antigenetically, there are four distinct serotypes of the dengue virus: DENV1, DENV2, DENV3, and DENV4. Each serotype has several genotypes or subtypes. Although there are different serotype, but in human only one serotype induce lifelong immunity against particular serotype re-infection but only partial and temporary immunity against the other serotypes ${ }^{1}$ and ${ }^{2}$. Every serotype has unique characteristics and shows harsh manifestation in particular population depending on interaction with response of host. Several preventive measures have taken The World Health Organization (WHO) to control the spread of dengue virus infection. However in several parts of the world still new outbreaks were reported during post monsoon season. Better education, public awareness programs, newer diagnostic techniques and proper monitoring of vector control are required to prevent outbreaks.

In the microbiology laboratory, diagnostic methods commonly used for confirming dengue infection involve as detection of virus antigen in serum or plasma, virus isolation and also detection of dengue virusspecific antibodies in serum and other body fluids. Molecular techniques are more expensive so most of laboratories favor detection of $\operatorname{IgM}, \operatorname{IgG}$, and NS1Ag by ELISA as confirmatory test which is also recommended by the WHO. Many studies show high prevalence of dengue among the population from different parts of the developing countries. ${ }^{3-7}$ Dengue disease is first isolated in Japan in 1942. ${ }^{8}$ Primary infection results acute febrile illness known as Dengue Fever (DF) which usually clear by an immune response complex in about 7 days. Secondary infection is more severe which results in Dengue Shock Syndrome (DSS) or hemorrhagic fever (DHF) or both. This can be life-threatening and may lead to death. ${ }^{9}$ The fatality rate is $1-5 \% .^{10}$

Last few decades, in India dengue have dramatically expanded with rapidly changing epidemiology. ${ }^{11}$ In India epidemic of dengue virus was in $1996 .{ }^{12}$ In 2003 all four serotypes of dengue viruses were found. ${ }^{2}$ In the years 2005-2008, outbreaks of DF/DHF were reported from various states in India. ${ }^{13}$ in 2010 India reported a total of 28,292 cases and 110 deaths, the highest number of cases and number deaths in a single year in the country in the previous two decades. ${ }^{14}$ This paper tried to review current perspectives of dengue infection among population of Bhilai visiting tertiary health institution. In study also we have compared the serological profiles of the dengue cases.

\section{MATERIALS AND METHODS}

The present study was conducted in CCMMC and hospital, a tertiary healthcare centre, catering to the healthcare needs of the people in and around Krud village, Bhilai, Durg district of Chhattisgarh. The samples received were performing retrospective analysis in the department of microbiology and process in the departmental microbiology laboratory obtaining during monsoon season, over a period of 3 months from august 2018 to October 2018. Individual visiting to hospital of all age groups, clinically suspected of dengue by presented 1-9 days of fever were include in this study. The serum was separated from the sample and treat with early dengue diagnostic tests, viz., rapid NS1 antigen, IgG and IgM. The duration of fever (in days) and other relevant clinical information were recorded from the requisition form. Rapid test for dengue as NS1Ag, $\operatorname{IgG}$ and IgM test were performed in accordance with the manufacturer's instructions. The dengue NS1 Ag, IgG and IgM rapid test is an in vitro immunochromatographic test (ICT) which is a onestep assay designed for the qualitative determination of dengue $\mathrm{NS} 1 \mathrm{Ag}$, IgG and IgM in human serum for the diagnosis dengue infection. About $50 \mu \mathrm{l}$ of patients' serum was added to the sample well marked as " $\mathrm{S}$ " and then three drops of buffer were added in the buffer well marked as "B". The result was interpreted in 20-30 min. The presence of only one color line within the control line marked as " $\mathrm{C}$ " indicated negative result and the presence of two color lines within the marked as " $\mathrm{T}$ " band and " $\mathrm{C}$ " line indicated a positive result. The test was concluded as invalid when no control line (C) was found. However the positive samples were also processed for ELISA as a confirmatory test.

\section{RESULT}

During the study period august 2018 to October 2018, total 1308 serum samples were collected from suspected Dengue fever patients. Among 1308 samples 412(31.5\%) samples were found to be positive dengue fever. Month -wise distribution of dengue positive cases over 3 months period as in August $41.73 \%$, in September $21.45 \%$ and in October $22.88 \%$ were positive as shown in Table 1 below. In the month of August maximum number of sample were received.

Out of 1308 patients with dengue suspected patients $792(60.6 \%)$ were male and 516(39.4\%) were female. Among $60.6 \%$ of male $28.2 \%$ were positive and out of $39.4 \%$ of female $36.6 \%$ were positive for dengue. Although male patient were more in number but female showed more prevalence to dengue fever as shown in Table 2 below. 


\begin{tabular}{lccc}
\multicolumn{4}{l}{ Table 1: Showing total no. of patients with } \\
positive cases within $\mathbf{3}$ months \\
\hline Month & $\begin{array}{c}\text { Total number } \\
\text { of patients }\end{array}$ & $\begin{array}{c}\text { Number of } \\
\text { positive patients }\end{array}$ & Percentage \\
\hline Aug & 623 & 260 & 41.73 \\
Sept & 331 & 71 & 21.45 \\
Oct & 354 & 81 & 22.88 \\
Total & 1308 & 412 & 31.50 \\
\hline
\end{tabular}

\begin{tabular}{|c|c|c|c|c|}
\hline Sex & $\begin{array}{c}\text { Total number } \\
\text { of patients }\end{array}$ & Percentage & Positive & Percentage \\
\hline Male & 792 & 60.6 & 223 & 28.2 \\
\hline Female & 516 & 39.4 & 189 & 36.6 \\
\hline Total & 1308 & 100 & 412 & 31.5 \\
\hline
\end{tabular}

\begin{tabular}{lccc}
\multicolumn{4}{l}{ Table 3: Age profile of the patients } \\
\hline $\begin{array}{l}\text { Age } \\
\text { group }\end{array}$ & $\begin{array}{c}\text { Total number } \\
\text { of patients }\end{array}$ & $\begin{array}{c}\text { Number of } \\
\text { positive patients }\end{array}$ & Percentage \\
\hline $01-10$ & 156 & 32 & 20.51 \\
$10-20$ & 120 & 67 & 55.83 \\
$20-30$ & 383 & 126 & 32.90 \\
$30-40$ & 226 & 92 & 40.71 \\
$40-50$ & 126 & 52 & 41.27 \\
$50-60$ & 145 & 26 & 17.93 \\
$60-70$ & 95 & 12 & 12.63 \\
$70-80$ & 57 & 5 & 8.77 \\
Total & 1308 & 412 & 31.50 \\
\hline
\end{tabular}

In this study from the age between 1 year old to 80 years old patients were included. The mean age was $33.82 \pm 11.17$ years old and Standard deviation $\sigma$ is 18.9063. Age between 10-20 years old (55.83\%) patients were most affected age group and 70-80 years old $(8.77 \%)$ age group were least affected as shown in Table 3 below.

Out of 1308 samples 412 samples were positive for one or more Dengue specific parameters as shown in Table 4 below. Out of total 412 positive samples NS1 Ag and IgG $\mathrm{Ag}$ only $(23.3 \%)$ are maximum in number whereas least positive were NS1 Ag and IgM Ag only 9.5\%.

In this study among the dengue positive patients, dengue parameter associated with thrombocytopenia was also recorded as shown in Table 5 below. Out of total positive cases thrombocytopenia was seen in $207(50.2 \%)$ patients. In 213 NS1 Ag positive patients thrombocytopenia was seen in $116(54.5 \%)$ whereas considering antibodies along thrombocytopenia was observed in $91(45.7 \%)$ out of 199 patients. The $\mathrm{p}=0.307$ which showed that there was no significant difference between the above two parameters in relation to thrombocytopenia. In 78 NS1 only positive patients

\begin{tabular}{lcc}
$\begin{array}{l}\text { Table 4: Comparison of various dengue } \\
\text { parameters }\end{array}$ \\
\hline Parameters & Number & Percentage \\
\hline NS1 only & 78 & 18.9 \\
IgM only & 74 & 18.0 \\
IgG only & 68 & 16.5 \\
NS1 and IgM only & 39 & 9.5 \\
NS1 and IgG only & 96 & 23.3 \\
IgM and IgG only & 57 & 13.8 \\
Total & 412 & 100 \\
\hline
\end{tabular}

NS1: Non- structural protein 1, IgM: Immunoglobuline M, IgG: Immunoglobuline G

\begin{tabular}{|c|c|c|c|}
\hline Parameters & Number & $\begin{array}{l}\text { Platelet count } \\
<1,00,000 / \mathrm{ml}\end{array}$ & Percentage \\
\hline NS1 only & 78 & 37 & 47.4 \\
\hline IgM only & 74 & 32 & 43.2 \\
\hline IgG only & 68 & 27 & 39.7 \\
\hline NS1 and IgM only & 39 & 25 & 64.1 \\
\hline NS1 and IgG only & 96 & 54 & 56.3 \\
\hline IgM and IgG only & 57 & 32 & 56.1 \\
\hline Total & 412 & 207 & 50.2 \\
\hline
\end{tabular}

\begin{tabular}{|c|c|c|}
\hline Outcome & $\begin{array}{c}\text { Number of } \\
\text { dengue infection }\end{array}$ & Percentage \\
\hline Discharge patients & 361 & 87.6 \\
\hline Refer & 49 & 11.9 \\
\hline Death & 2 & 0.5 \\
\hline Total & 412 & 100 \\
\hline
\end{tabular}

$47.4 \%$ thrombocytopenia was observed whereas considering NS1 and IgM Ag only thrombocytopenia was observed in $25(64.1 \%)$ out of 39 positive patients. The $p=0.353$ which showed that there was no significant difference between the above two parameters in relation to thrombocytopenia.

For all the dengue positive patients was monitored closely following admission as shown in Table 6 below. After the admission of the patient's regular follow up was done as a result following outcomes were observed. Out of 412 positive patients 361 (87.6\%) discharge from the hospital after the recovery whereas $49(11.9 \%)$ were refer to higher government hospital centre and $2(0.5 \%)$ were death.

\section{DISCUSSION}

According to the World Health Organization, in the past 50 years globally the incidence of dengue has shot up 30-fold. Dengue disease presents highly complex 
pathophysiological, economic, and ecologic problems. ${ }^{15}$ In order to provide early information about management and public health control of dengue outbreaks, it is important to diagnosis of acute dengue infection on the first days of manifestation of the clinical symptoms. ${ }^{16}$ In dengue infection the role of NS1 Ag for early detection is currently being assess by many investigators, without the requirement of paired sera. ${ }^{17,18}$ The NS1 antigen is a highly specific marker of dengue infection which circulates uniformly in all serotypes of dengue virus. During the first few days of illness it circulates at high level as there is no cross reaction of the dengue NS1 protein, with those of other related flaviviruses. ${ }^{19,20}$ According to the studied of Kulkarni et al ${ }^{21}$ NS1 alone and with IgM correlated well with thrombocytopenia whereas in this study there was no correlation. This may be because level of NS1 depends on the viral load as level of NS1 is indirectly proportional to the duration of illness increases. After starting antibodies the NS1 antigen is sequestered into immune complexes. ${ }^{17}$

According to the study of Ghouth et $\mathrm{al}^{18}$ and Padhi S et al ${ }^{19}$ age group was between 11 - 30 years are commonly effected. Dengue cases was about to be $28.5 \%$ and $29.4 \%$ for the age groups 11-20 years and 21-30 years respectively which was lesser than this study. Sunyoto $\mathrm{T}$ et $\mathrm{al}^{20}$ studied $7 \%$ patients Dengue cases were reported which was hospitalized and no report of death among patients whereas in this study $0.5 \%$ of death were recorded. Annually about $5 \%$ of the cases require hospitalization worldwide. However in India has seen an increase in the number of cases over the years, mortality is decreasing. ${ }^{21}$

\section{CONCLUSION}

In dengue positive patients the association of thrombocytopenia was highly significant as compared to thrombocytopenia in dengue negative patients. Especially in developing countries like India where there are poor resources for diagnosis of dengue infection, the sensitivity of these tests is more than immunochromatographic.

Test (ICT). By NS1 assay early detection of dengue infection can help in early confirmation and management of this before its gets complicate. National level of study from different places should be estimate for true burden of dengue in India. From various sectors for the prevention of dengue policy making and health education should be provided to control further disease transmission. Therefore commercial available dengue NS1 antigen test kits provided additional laboratory diagnostic tool for early detection of dengue.

\section{REFERENCES}

1. Gubler DJ. The changing epidemiology of yellow fever and dengue, 1900 to 2003: full circle? Comp Immunol Microbiol Infect Dis 2004; 27: 319-330.

2. Dar L, Gupta E, Narang P and Broor S. Cocirculation of dengue serotypes, Delhi, India. Emerg Infect Dis 2006; 12:352-353.

3. Dar L, Broor S, Sengupta S, Xess I and Seth P. The first major outbreak of dengue hemorrhagic fever in Delhi, India. Emerg Infect Dis 1999; 5:589-590.

4. Agarwal R, Kapoor S, Nagar R, Misra A, Tandon R, Mathur A, et al. A clinical study of the patients with dengue hemorrhagic fever during the epidemic of 1996 at Lucknow, India. Southeast Asian J Trop Med Public Health 1999; 30:735-740.

5. Gupta E, Mohan S, Bajpai M, Choudhary A and Singh G. Circulation of dengue virus1 (DENV1) serotype in Delhi, during 201011 after dengue virus3 (DENV3) predominance: A single centre hospitalbased study. J Vector Borne Dis 2012; 49:82-85

6. Ahmed NH and Broor S. Dengue fever outbreak in Delhi, North India: A clinicoepidemiological study. Indian J Community Med 2015; 40:135-138.

7. Mishra G, Jain A, Prakash O, Prakash S, Kumar R, Garg RK, et al. Molecular characterization of dengue viruses circulating during 2009-2012 in Uttar Pradesh, India J Med Virol 2015;87:68-75.

8. Simmons CP, Farrar JJ, van Vinh Chau N and Wills B. Dengue. New England Journal of Medicine 2012;366(15):1423-1432. https://doi.org/10.1056/NEJMra1110265

9. Guzman MG and Kouri G. Dengue: An update. The Lancet infectious diseases 2011; 2(1):, 33- 42. https://doi.org/10.1016/ S1473-3099(01)00171-2

10. Ranjit $S$ and Kissoon N. Dengue hemorrhagic fever and shock syndromes. Pediatric Critical Care Medicine 2011; 12(1), 90-100.

11. Chakravarti A, Matlani M, Kashyap B and Kumar A. Awareness of changing trends in epidemiology of dengue fever is essential for epidemiological surveillance. Indian J Med Res 2012; 30:222-226.

12. Broor S, Dar L, Sengupta S, Chakaraborty M, Wali JP, Biswas A, et al. Recent dengue epidemic in Delhi, India. In Factors in the emergence of arbovirus diseases Edited by: Saluzzo JE, Dodet B. Paris: Elsevier; 1997: P123-127.

13. Dash AP, Bhatia R and Kalra NL. Dengue in South-East Asia: an appraisal of case management and vector control. In: World Health Organization, Dengue Bulletin, volume 36. Geneva: World Health Organization; 2012: 1-13. Available from: http:// www.wpro.who.int/mvp/ epidemiology/dengue/Dengue_ Bulletin_Vol36.pdf. Accessed August 29, 2014.

14. Gupta E, Mohan S, Bajpai M, Choudhary A and Singh G. Circulation of Dengue virus-1 (DENV-1) serotype in Delhi, during 2010-11 after Dengue virus-3 (DENV-3) predominance: a single centre hospital-based study. J Vector Borne Dis 2012; 49:82-85.

15. Gubler DJ. The economic burden of dengue. Am J Trop Med Hyg 2012; 86:743-744.

16. Kumarasamy V, Chua SK, Hassan Z, Wahab AH, Chem YK, Mohamad $\mathrm{M}$, et al. Evaluating the sensitivity of a commercial dengue NS1 antigen-capture ELISA for early diagnosis of acute dengue virus infection. Singapore Med J 2007; 48:669-673.

17. Hang VT, Nguyet NM, Trung DT, Tricou V, Yoksan S, Dung NM, et al. Diagnostic accuracy of NS1 ELISA and lateral flow rapid tests for dengue sensitivity, specificity and relationship to viraemia and antibody responses. PLoS Negl Trop Dis 2009;3:360.

18. Ghouth AS Bin, Amarasinghe A and William LG. Dengue outbreak in Hadramout, Yemen, 2010: An epidemiological perspective. Am J Trop Med Hyg 2012; 86(6):1072-1076.

19. Sanghamitra $P$, Muktikesh D, Pritilata $P$, Banojini $P$, Indrani $M$ 
and Susmita S, et al. A three year retrospective study on the increasing trend in seroprevalence of dengue infection from southern Odisha, India.

20. Sunyoto T, Bhatia R and Dash AP. Changing epidemiology of dengue in South-East Asia. WHO South-East Asia J Public Heal 2013;2(1):23. Doi: 10.4103/2224-3151.115830.

21. Cecilia D. Current status of dengue and chikungunya in India. WHO South-East Asia J Public Heal 2014;3(1).

\section{Authors Contribution:}

DHC- Concept, collected data and design of the study, reviewed the literature, literature search, manuscript preparation and critical revision of the manuscript.

Work attributed to:

Department of Microbiology, CCMMC \& Hospital, Durg. (C.G.).

Orcid ID:

Dr. Dhruba Hari Chandi - (1) http://orchid.org/0000-0002-7749-6839

Source of Support: Nil, Conflict of Interest: None declared. 